\title{
Wave motion analysis of finite coupled plates with Reverberation Ray Matrix Method
}

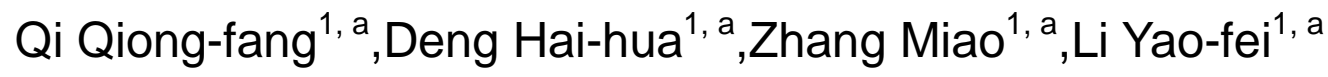 \\ ${ }^{1}$ The Second Ship Design Institute of Wuhan, Wuhan 430064, China \\ a15072416674@163.com
}

\begin{abstract}
Key words: Vibration; coupled plate; reverberation ray matrix method; loss factor; active power flow; reactive power flow.

Abstract. The purpose is to analyze the vibrational wave motion of finite coupled plates considering boundary conditions. Since reverberation ray matrix is a semi-analytical method, Local dual coordinate of each discrete plate was introduced to reverberation ray matrix method. The vibrational formulas of coupled plates were deduced by current reverberation ray matrix. The result of reverberation ray matrix was compared with the result of finite element method. The correctness of the derivation of wave solution was verified. In addition, the high precision of self-complied program of MATLAB based on reverberation ray matrix was verified. The calculation time of reverberation ray matrix and finite element method were compared and the high efficiency of solution was validated. The influence of loss factor on active power and reactive power flows was analyzed. It demonstrated that the loss factor has heavy influence on the active power flow and has little effect on the reactive power flow away from the resonance frequency. At the resonance frequency, the increase of the loss factor could effectively reduce the peak value of the reactive power flow. Loss factor have the opposite effect on power flow at resonance region and non-resonant region.
\end{abstract}

\section{Introduction}

During the operation of the ship, its power equipment will inevitably produce vibration and noise, which has become a significant topic and attracts many researchers' attention. International Maritime Organization and the Equipment Committee have new demands on ship noise, higher requirements of the noise of ship cabin and the sound insulation index of bulkheads and decks are put forward. Most of the vibration produced by power equipment will be transferred from the pedestal to the hull directly and become structural-born sound. The wave transmission characteristic of vibrations from the source to adjacent plates should be analyzed.

In 1998, PAO, et al. [1] proposed Reverberation Ray Matrix Method (MRRM), assuming that the elastic wave propagated in the structure and scattered at the node, the scattering matrix was obtained according to the equilibrium equation, and the return wave was obtained according to the scattering matrix and the phase matrix. At present, Reverberation Ray Matrix Method is mainly used to analyze the vibration response of beams and frames. Cuschieri J M, et al. [2] employed power flow to research the L-shaped plate, when the flexural wave number multiplied by the thickness was less than 0.1 , the in-plane wave plate could be ignored. Wave power flow and flexural wave power flow of finite L-shaped plate were researched by Kessissoglou, et al. [3-4]. Wave method results show that in-plane wave of L-shaped plate can be converted into out-plane wave through the connected line. Miao F X, et al. [5-6] employed MRRM to analyze the modal of the plane frame and the dynamic response of the classical laminated composite beam, the first - order shear composite beam and the laminated composite frame. Jiang J Q, et al [7] used MRRM and transfer matrix method to study the Euler - Bernoulli characteristics of beams, the numerical stability was verified by the comparison of natural frequency. Liu C C, et al. [8-10] employed MRRM to analyze the short-term transient response of finite stiffened plates and cylindrical shells under impact loads. Comparing the analytical solutions with the results of the model tests, it was shown that the MRRM was an effective method for short transient response.

In this paper, the local dual coordinates were introduced based on MRRM, the coupled plate 
was discretized at the online connection, the local dual coordinate system of each discrete plate was established. The in-plane wave and the out-plane wave amplitude coefficient of each discrete plates are discretized. The force and displacement state vector response and vibration formula of each discrete plate were deduced when the coupled plate were under the point excitation. The vibration power flow of the structure was calculated by MATLAB program, and the efficiency and validity of MRRM were verified by comparison with the finite element numerical calculation. The influence of structural loss factor on active power flow and passive power flow was discussed.

\section{Theoretical formulations}

Fig. 1 illustrated the coupled plate with the employed global coordinate. The plates are discrete at the place of the excitation force and the connection line. The connected angle is an arbitrary angle $\beta$. The connected lines of discrete plates are numbered by $0,1,2,3$. The discrete plates are numbered by plate 01, plate 12 and plate 23. Each discrete plate contained a local dual coordinate system, such as, the coordinate system of left edge of the plate 01 was $\mathrm{x}_{01} \mathrm{y}_{01} \mathrm{z}_{01}$, the right was $\mathrm{x}_{10} \mathrm{y}_{10} \mathrm{z}_{10}$. The origin of the right end coordinate of the plate 01 coincides with of the left end of the plate 12. The displacement vector of each discrete plate was $u, v, w$. The force vector was Nxx, Nxy, Vxy, respectively.

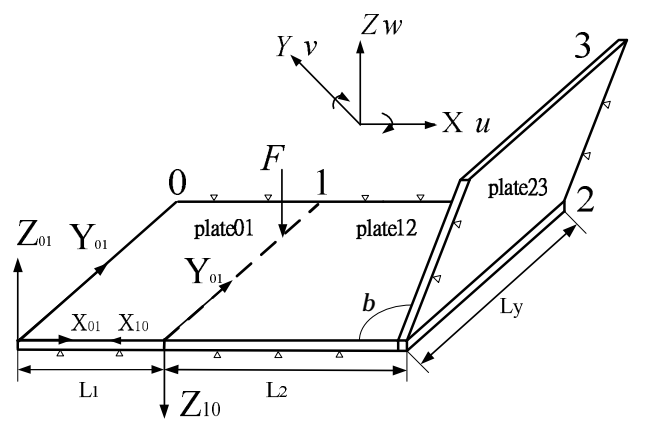

Fig. 1 Diagram of coupled plate with connected angle

Wave solutions of bending motion of coupled plate. Two parallel edges of coupled plates are simply supported along $y$ direction. The length along $x$ direction is $L_{1}, L_{2}, L_{3}$, respectively. The drive force in local coordinate is $\left(x_{0}, y_{0}\right)$. Each rectangular plate is considered to have bending motion and in-plane motion. The plate is assumed to have small deformation, so the in-plane and out-plane motion are independent. Based on vibration control theory of plate, bending differential equations are expressed as:

$$
\begin{aligned}
\kappa G h\left(\nabla^{2}+\frac{\partial \varphi_{x}}{\partial x}+\right. & \left.\frac{\partial \varphi_{y}}{\partial y}\right)+f(x, y, t)=\rho h \frac{\partial^{2} w}{\partial t^{2}} \\
& \frac{D}{2}\left[(1-\mu) \nabla^{2} \varphi_{x}+(1+\mu) \frac{\partial}{\partial x}\left(\frac{\partial \varphi_{x}}{\partial x}+\frac{\partial \varphi_{y}}{\partial y}\right)\right]-\kappa G h\left(\varphi_{x}+\frac{\partial w}{\partial x}\right)=\frac{\rho h^{3}}{12} \frac{\partial^{2} \varphi_{x}}{\partial t^{2}} \\
& \frac{D}{2}\left[(1-\mu) \nabla^{2} \varphi_{y}+(1+\mu) \frac{\partial}{\partial y}\left(\frac{\partial \varphi_{x}}{\partial x}+\frac{\partial \varphi_{y}}{\partial y}\right)\right]-\kappa G h\left(\varphi_{y}+\frac{\partial w}{\partial y}\right)=\frac{\rho h^{3}}{12} \frac{\partial^{2} \varphi_{y}}{\partial t^{2}}
\end{aligned}
$$

Two parallel edges of rectangular plate are simple supported, the displacement:

$$
\begin{aligned}
w & =\sum_{n=1}^{\infty}\left(a_{1 n} e^{\lambda_{1} x}+d_{1 n} e^{-\lambda_{1} x}+a_{2 n} e^{\lambda_{2} x}+d_{2 n} e^{-\lambda_{2} x}\right) \sin \left(k_{y} y\right) e^{i \omega t} \\
\varphi_{x} & \left.=\sum_{n=1}^{\infty}\left[\left(\sigma_{1}-1\right) \lambda_{1}\left(a_{1 n} e^{\lambda_{1} x}-d_{1 n} e^{-\lambda_{1} x}\right)+\left(\sigma_{2}-1\right) \lambda_{2}\left(a_{2 n} e^{\lambda_{2} x}-d_{2 n} e^{-\lambda_{2} x}\right)-k_{y} a_{3 n} e^{\lambda_{3} x}-k_{y} d_{3 n} e^{-\lambda_{3} x}\right)\right] \sin \left(k_{y} y\right) e^{i \omega t} \\
\varphi_{y} & =\sum_{n=1}^{\infty}\left[\left(\sigma_{1}-1\right) k_{y}\left(a_{1 n} e_{1}^{\lambda_{1} x}+d_{1 n} e^{-\lambda_{1} x}\right)+\left(\sigma_{2}-1\right) k_{y}\left(a_{2 n} e^{\lambda_{2} x}+d_{2 n} e^{-\lambda_{2} x}\right)-\lambda_{3} a_{3 n} e^{\lambda_{3} x}+\lambda_{3} d_{3 n} e^{-\lambda_{3} x}\right] \cos \left(k_{y} y\right) e^{i \omega t}
\end{aligned}
$$

Where $u$ is the longitudinal displacement, $v$ is the $y$-direction, $w$ is the lateral displacement, $\varphi_{x}$ is around the $\mathrm{y}$-axis angle, $\varphi_{y}$ is around the $x$-axis angle. The $E, h, \mu, \kappa$ indicates the Young's modulus, plate thickness, Poisson ratio, shear factor, respectively. $f(x, y, t)$ is the external 
excitation. $k_{y}$ is the wave number along $y$ direction. $m$ is the modal number along $y$ direction. $a_{1 n}, a_{2 n}, a_{3 n}, a_{4 n}, a_{5 n}$ is respectively the amplitude of the arrival wave. $d_{1 n}, d_{2 n}, d_{3 n}, d_{4 n}, d_{5 n}$ is respectively the amplitude of the wave. $\lambda_{1} 、 \lambda_{2} 、 \lambda_{3}$ are the three-eigenvalues of the volatility of the out-of-plane bending motion, respectively. $k_{1,2}^{2}$ is the corresponding three elastic waves, respectively. The middle parameter is $\sigma_{1,2}$, respectively. The time item of $e^{i \omega t}$ above equation was omitted.

Wave solutions of in-plane motion of coupled plates. In-plane equation of vibration control can be rewritten as:

$$
\begin{gathered}
\frac{E h}{1-v^{2}}\left(\frac{\partial^{2} u}{\partial x^{2}}+\frac{1-v}{2} \frac{\partial^{2} u}{\partial y^{2}}+\frac{1+v}{2} \frac{\partial^{2} v}{\partial x \partial y}\right)=\rho h \frac{\partial^{2} u}{\partial t^{2}} \\
\frac{E h}{1-v^{2}}\left(\frac{\partial^{2} v}{\partial x^{2}}+\frac{1-v}{2} \frac{\partial^{2} v}{\partial y^{2}}+\frac{1+v}{2} \frac{\partial^{2} u}{\partial x \partial y}\right)=\rho h \frac{\partial^{2} v}{\partial t^{2}}
\end{gathered}
$$

In-plane displacement can be expressed as:

$$
\begin{gathered}
u=\sum_{n=1}^{\infty}\left(\lambda_{4} a_{4 n} e^{\lambda_{4} x}-\lambda_{4} d_{4 n} e^{-\lambda_{4} x}+k_{y} a_{5 n} e^{\lambda_{5} x}+k_{y} d_{5 n} e^{-\lambda_{5} x}\right) \sin \left(k_{y} y\right) e^{i \omega t} \\
v=\sum_{n=1}^{\infty}\left(k_{y} a_{4 n} e^{\lambda_{4} x}+k_{y} d_{4 n} e^{-\lambda_{4} x}+\lambda_{5} a_{5 n} e^{\lambda_{5} x}-\lambda_{5} d_{5 n} e^{-\lambda_{5} x}\right) \cos \left(k_{y} y\right) e^{i \omega t}
\end{gathered}
$$

Where $k_{L}$ is the longitudinal wave number, $k_{S}$ is expressed as the number of shear wave. The two eigenvalues of in-plane wave are $\lambda_{4}=\sqrt{k_{y}^{2}-k_{L}^{2}} 、 \lambda_{5}=\sqrt{k_{y}^{2}-k_{s}^{2}}$.

The following relationship should be satisfied by force and displacement [4].

$$
\begin{aligned}
& M_{x x}=D\left(\frac{\partial \varphi_{x}}{\partial x}+\mu \frac{\partial \varphi_{y}}{\partial y}\right), M_{x y}=\frac{1-\mu}{2} D\left(\frac{\partial \varphi_{x}}{\partial y}+\frac{\partial \varphi_{y}}{\partial x}\right), V_{x}=\kappa G h\left(\frac{\partial w}{\partial x}+\varphi_{x}\right), \\
& N_{x x}=\frac{E h}{1-\mu^{2}} \frac{\partial u}{\partial x}+\mu \frac{E h}{1-\mu^{2}} \frac{\partial v}{\partial y}, N_{x y}=\frac{E h}{2(1+\mu)}\left(\frac{\partial u}{\partial x}+\frac{\partial v}{\partial y}\right)
\end{aligned}
$$

Where $M_{x x}, V_{x}, M_{x y}$ indicates the bending moment, shear force, torque, respectively. $V_{x}, N_{x x}$, $N_{x y}$ is the out-plane shear force, the in-plane axial force, the in-plane shear force, separately.

Boundary condition of coupled plate. According to MRRM, the displacement vector and force vector of the discrete plate in modal $n$ can be rewritten, so Eqs. (4)-(6) and Eqs. (9)-(10) can be in the form of matrix as follows:

$$
\boldsymbol{W}_{n}=\boldsymbol{Y}_{n} \boldsymbol{A}_{n \delta} \boldsymbol{P}_{n}(-x) \boldsymbol{a}_{n}+\boldsymbol{Y}_{n} \boldsymbol{D}_{n \delta} \boldsymbol{P}_{n}(x) \boldsymbol{d}_{n}, \boldsymbol{F}_{n}=\boldsymbol{Y}_{n} \boldsymbol{A}_{n f} \boldsymbol{P}_{n}(-x) \boldsymbol{a}_{n}+\boldsymbol{Y}_{n} \boldsymbol{D}_{n f} \boldsymbol{P}_{n}(x) \boldsymbol{d}_{n}
$$

Where the displacement state matrix of the $n^{\text {th }}$ mode at the section of $x$ is $\boldsymbol{W}_{n}=\left\{\begin{array}{lllll}\varphi_{x n} & \varphi_{y n} & w_{n} & u_{n} & v_{n}\end{array}\right\}^{T} . \quad \boldsymbol{F}_{n}=\left\{\begin{array}{lllll}M_{x x n} & M_{x y n} & V_{x n} & N_{x n} & N_{x y n}\end{array}\right\}^{T}$ is force state matrix. $\boldsymbol{P}_{n}(x)=\operatorname{diag}\left\{e^{-\lambda_{1} x} \quad e^{-\lambda_{2} x} \quad e^{-\lambda_{3} x} \quad e^{-\lambda_{4} x} \quad e^{-\lambda_{5} x}\right\}$ indicating the elastic wave. Phase diagonal matrix $\boldsymbol{Y}_{n}=\operatorname{diag}\left\{\begin{array}{llllll}\sin k_{y} y & \cos k_{y} y & \sin k_{y} y & \sin k_{y} y & \cos k_{y} y\end{array}\right\} \quad$ is modal matrix along $y$ direction. $\boldsymbol{d}_{n}=\left\{\begin{array}{lllll}d_{1 n} & d_{2 n} & d_{3 n} & d_{4 n} & d_{5 n}\end{array}\right\}^{T}$ is the coefficient matrix of amplitude of departing wave. $\boldsymbol{a}_{n}=\left\{\begin{array}{lllll}a_{1 n} & a_{2 n} & a_{3 n} & a_{4 n} & a_{5 n}\end{array}\right\}^{T}$ is the coefficient matrix of amplitude of arriving wave. $\boldsymbol{A}_{n \delta}, \boldsymbol{A}_{n f}, \boldsymbol{D}_{n \delta}, \boldsymbol{D}_{n f}$ can be derived from arriving wave and departing wave.

The right edge parallel to the $y$ direction of the plate 01 is connected to the left edge of the plate 12 at line 1 , which should meet continuity equations as follows:

$$
\begin{aligned}
& \left.\varphi_{x n}^{01}\right|_{x=l^{0^{1}}}=\left.\varphi_{x n}^{12}\right|_{x=0},\left.\varphi_{y n}^{01}\right|_{x=l^{0^{11}}}=-\left.\varphi_{y n}^{12}\right|_{x=0},\left.w_{n}^{01}\right|_{x=l^{01}}=-\left.w_{n}^{12}\right|_{x=0},\left.u_{n}^{01}\right|_{x=l^{01}}=-\left.u_{n}^{12}\right|_{x=0},\left.v_{n}^{01}\right|_{x=l^{01}}=\left.v_{n}^{12}\right|_{x=0},
\end{aligned}
$$

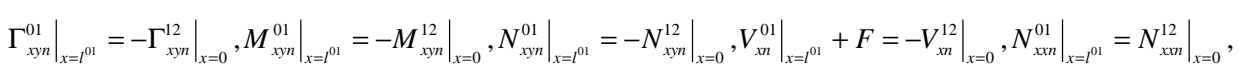

The right edge parallel to the $y$ direction of the plate 12 is connected to the left edge of the plate 23 through the line 2 , and the continuity conditions are defined as: 


$$
\begin{array}{r}
\left.w_{x n}^{12}\right|_{x=l^{12}}=\left.w_{x n}^{23} \cos \beta\right|_{x=0}-\left.u_{x n}^{23} \sin \beta\right|_{x=0},\left.u_{x n}^{12}\right|_{x=l^{12}}=\left.w_{x n}^{23} \sin \beta\right|_{x=0}+\left.u_{x n}^{23} \cos \beta\right|_{x=0},\left.v_{x n}^{12}\right|_{x=l^{12}}=\left.v_{x n}^{23}\right|_{x=0}, \\
\left.M_{x n}^{12}\right|_{x=l^{12}}=-\left.M_{x x}^{23}\right|_{x=0},\left.\Gamma_{x y n}^{12}\right|_{x=l^{12}}=-\left.\varphi_{y n}^{23} \sin \beta\right|_{x=0}+\left.\Gamma_{x y n}^{23} \cos \beta\right|_{x=0},\left.V_{x n}^{12}\right|_{x=l^{12}}=-\left.V_{x n}^{23} \cos \beta\right|_{x=0}+\left.N_{x n}^{23} \sin \beta\right|_{x=0}, \\
\left.N_{x n}^{12}\right|_{x=l^{12}}=-\left.V_{x n}^{23} \sin \beta\right|_{x=0}-\left.N_{x n}^{23} \cos \beta\right|_{x=0},\left.N_{x y n}^{12}\right|_{x=l^{12}}=-\left.N_{x y n}^{23}\right|_{x=0},\left.\varphi_{x n}^{12}\right|_{x=l^{12}}=\left.\varphi_{x n}^{23}\right|_{x=0}, \\
\left.\varphi_{y n}^{12}\right|_{x=l^{12}}=\left.\varphi_{y n}^{23} \cos \beta\right|_{x=0}+\left.\Gamma_{x y n}^{23} \sin \beta\right|_{x=0},
\end{array}
$$

If the two edge parallel to the $y$ direction of the plate 34 is simply supported, The boundary conditions are defined as:

$$
\left.N_{x x}^{34}\right|_{x=l^{34}}=0,\left.v^{34}\right|_{x=l^{34}}=0,\left.w^{34}\right|_{x=l^{34}}=0,\left.M_{x x}^{34}\right|_{x=l^{34}}=0,
$$

If the right edge parallel to the $y$ direction of the plate 34 is free, similar to the boundary conditions, the corresponding balance equations had been omitted to avoid duplication. Each discrete plate has 10 unknown wave coefficients, there are three discrete plates, resulting in a total of 30 unknown amplitude coefficients. The connection line 0 and line 4 have five boundary conditions each, the connection line 1,2, 3 has 10 balance equations, respectively. The coupled plate under point excitation has a total of 30 unknown wave amplitude coefficients. There 30 balance equations, so the unknown coefficient could be solved. Then the displacement and internal force response were obtained.

The balance equations can be assembled in the following form, when $\mathbf{J}$ is $0,1,2,3$, the amplitude of arriving wave of the plate is $\boldsymbol{a}_{\boldsymbol{n}}$. The amplitude coefficients of departing wave of the plate is $\boldsymbol{d}_{n}$. The scattering matrix is $S_{n}$. Based on the discrete plate 01, the departing wave of the connection line 1 is $\boldsymbol{d}^{1}=\left[\begin{array}{ll}\boldsymbol{d}^{10} & \boldsymbol{d}^{01}\end{array}\right]^{T}$, the arriving wave and the arriving wave satisfied the following phase relationship:

$$
\left\{\begin{array}{l}
\boldsymbol{a}_{n}^{01} \\
\boldsymbol{a}_{n}^{10}
\end{array}\right\}=\left[\begin{array}{cc}
\boldsymbol{P}_{n}^{01}\left(x_{0}\right) & 0 \\
0 & \boldsymbol{P}_{n}^{10}\left(x_{0}\right)
\end{array}\right] \boldsymbol{U}_{\text {ele }}\left\{\begin{array}{l}
\boldsymbol{d}_{n}^{10} \\
\boldsymbol{d}_{n}^{01}
\end{array}\right\}, \boldsymbol{d}_{n}^{J}=\boldsymbol{S}^{J} \boldsymbol{a}_{n}^{J}
$$

Where $\boldsymbol{I}=\operatorname{diag}\left[\begin{array}{lllll}1 & 1 & 1 & 1 & 1\end{array}\right]$ represents the diagonal matrix, $\boldsymbol{U}_{\text {ele }}$ represents the cell unit of diagonal matrix. For any connection line $\mathrm{J}$, the scattering matrix of the arriving and departing waves could be established.

\section{Verification and discussions}

(a)

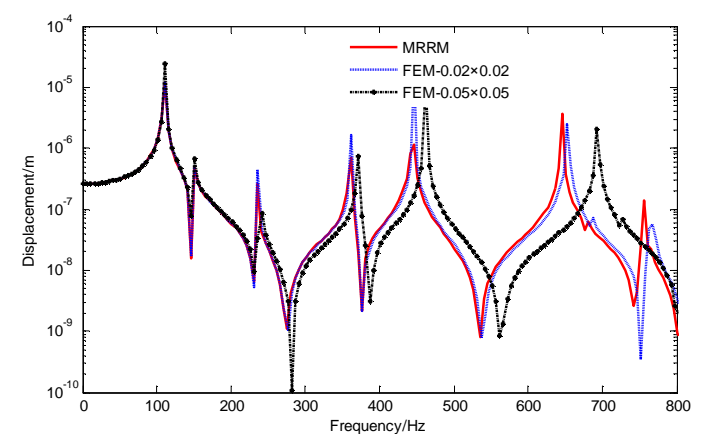

(b)

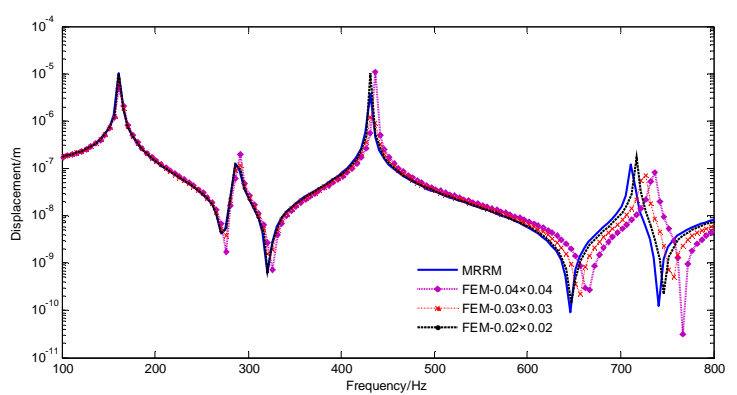

Fig. 2 Comparisons of transverse displacement response (a) connected angle $\beta=90$ degree (b) L-shaped plate when connected angle $\beta=90$ degree.

In order to verify the correctness of the formula of the coupled plate, MATLAB is used to calculate the dynamic response results of the MRRM and the finite element method are compared. For coupled plates, the properties of the plates are given as follows: length $L_{1}=L_{2}=L_{3}=0.4 \mathrm{~m}$, width $L_{y}=0.6 \mathrm{~m}$, thickness $h=10 \mathrm{~mm}$, Young's modulus of elasticity $E=2.1 \times 10^{11} \mathrm{~Pa}$, Poisson's ratio $\mu=0.3$ and density $\rho=7800 \mathrm{~kg} / \mathrm{m}^{3}$. The generalized inverse method of the MRRM and the frequency response of the finite element method at the excitation point is described Fig. 2(a). The excitation point is at middle of the line 1. The finite element size are $0.02 \mathrm{mÕ} 0.02 \mathrm{~m}$ and $0.05 \mathrm{mÕ} 0.05 \mathrm{~m}$. Frequency range of measurement is selected as $0 \mathrm{~Hz} \sim 800 \mathrm{~Hz}$ and frequency step is selected as $2 \mathrm{~Hz}$. The frequency responses of the Finite Element Method are in good agreement with the present 
MRRM. As the calculated frequency increases, the peak and phase of the curves are different, which are related to mesh density of Finite Element Method. Dynamic responses calculated by the present method are in good agreement with FEM, which proves that the derivation of wave solution of MRRM is correct.

The properties of responding model of Fig. 2(b) is described as: the length $L_{1}=L_{2}=L_{3}=0.8 \mathrm{~m}$, width $L_{y}=0.8 \mathrm{~m}$, thickness $h=8 \mathrm{~mm}$. Steel properties are in consistent with the model of Fig.2. The two edges parallel to the $x$ direction of each discrete plate are simply supported. The excitation force was located at the midpoint of the right end of plate 01, the transverse displacement was calculated at the excitation point of the L-shaped plate when connected angle was 90 degree. The Semi-analytical solution of MRRM was compared with the numerical results of finite element method. The model of finite element method of three kinds of mesh density is as follows: 0.02 mÕ 0.02 m, 0.03 mÕ 0.03 m, 0.04 mÕo 0.04 m.

As shown in Fig. 2, with the increase of mesh density, the displacement response curve regarding frequency of the finite element method approached to the result of MRRM, showing that the result of the Reverberation Wave Method was in high accuracy. Results of finite element method are different when the model with different mesh density. To meet the calculation accuracy in structural dynamic analysis with finite element method, the element length should be less than 1/6 of the minimum length of bending wave. For the coupled plate with connected angle $\beta=90$ degree, the corresponding minimum wavelength was $0.2 \mathrm{~m}$. When the upper limit of the calculated frequency was $800 \mathrm{~Hz}$, the element length should be less than $0.05 \mathrm{~m}$ to fulfill the precision requirement.

The calculation time of MRMM and finite element method with different mesh density were described in Tab. 2. The calculation results show that the calculation time increase obviously with the increase of mesh density of finite element method. The generalized inverse of the MRRM can reach the computational accuracy of the finite element method with high mesh density, and the solution time was shorter. MRRM is a semi-analytical method, according to the boundary condition and the vibration control equations, the unknown parameters can be directly calculated. For finite element method, a discrete element occupies a matrix storage space. It is not necessary to divide the coupled plates into smaller discrete plates for MRRM, which greatly improves the computational efficiency. At the same time, MRRM avoids modal instability when solving the dynamic response in high frequency range, and could effectively solve the problem in high frequency and could guarantee the accuracy of the calculation result. With three kinds of finite element mesh densities, the L-shaped plate had consumed 120s, 189s, 409s, respectively. With the number of discrete plates increase, the computation of finite element method takes time longer. For frequency response analysis, the current MRRM for coupled plates has the characteristics of high precision and high efficiency.

Table 1 Calculation time for different methods

\begin{tabular}{cc}
\hline Calculation Method & Calculate time consuming (seconds) \\
\hline FEM-0.02Õ 0.02 & 120 \\
FEM-0.03Õ 0.03 & 189 \\
FEM-0.04Õ 0.04 & 409 \\
MRRM & 5 \\
\hline
\end{tabular}

\section{Power flow analysis}

To have a clear understanding of vibration power transmission at the connected lines, time averaged intensity of vibration at a specified $x$ location are expressed, the real part of which is the active power flow [4].

$$
\left.I_{x}=-(1 \&)^{*} V_{x}+\left(\phi_{x}^{\&}\right)^{*} M_{x x}+(\phi \& y)\right)^{*} M_{x y}+(u \&)^{*} N_{x x}+(1)^{*} N_{x y}
$$

The power flow in the $x$ direction of a finite plate can be expressed as an integral of the $y$ direction intensity at the cross-section $x$. 
(a)

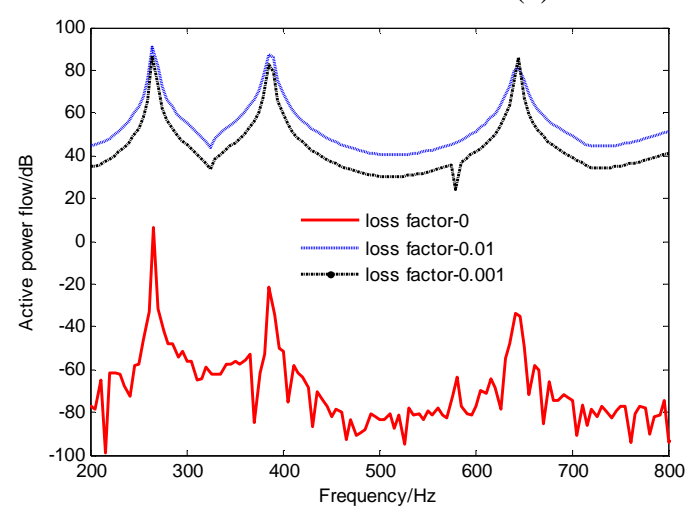

(b)
$P_{x}\left(\omega, x_{i}\right)=\int_{0}^{L_{y}} I_{x}\left(\omega, x_{i}\right) d y$

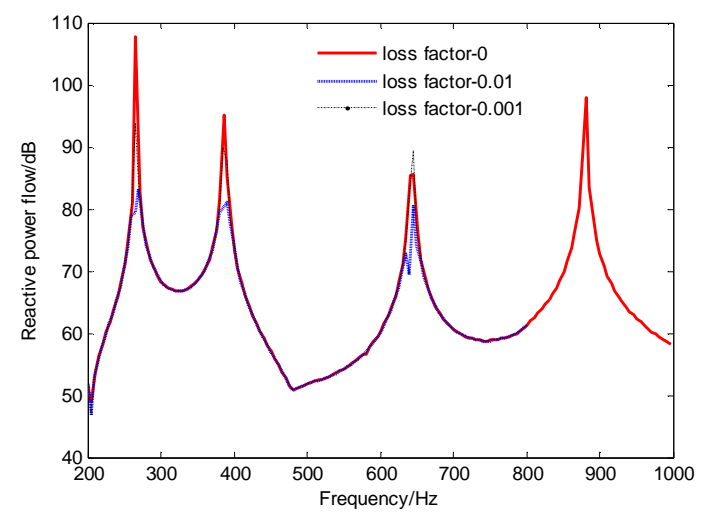

Fig. 3 Active and reactive power flow with different loss factor: (a) Active power flow with loss factor $\eta=0,0.1,0.001$. (b) Reactive power flow with loss factor $\eta=0,0.1,0.001$.

For coupled plates, the properties of the plates are given as follows: length $L_{1}=L_{2}=L_{3}=0.8 \mathrm{~m}$, width $L_{y}=0.8 \mathrm{~m}$, thickness $h=8 \mathrm{~mm}$, the connected angle $\beta=150^{\circ}$. The properties of responding model are in consistent with the model of Fig. 2. The calculated frequency range is $200-800 \mathrm{~Hz}$ and frequency step is $5 \mathrm{~Hz}$. The influence of loss factor on active power flow and reactive power flow was discussed. Fig. 4(a) presents the active power flow of coupled plate with the loss factor change. The comparison demonstrates that the loss factor is zero, the active power flow is much smaller than the active power flow is none-zero. The presence or absence of the loss factor has the significant impact on the active power flow. When the loss factor is zero, the active power flow is in the range of $-50 \mathrm{~dB}$ in the calculated frequency range.

As the loss factor increases, the active power flow is increased, and the loss factor does not have influence on active power flow at resonance frequency. It is demonstrated that active power flow is closely related to loss factor. When the loss factor is zero, the structural damping according to loss factor did not make the vibration energy less, the active power flow is small enough to be ignored. When the loss factor is non-zero, the vibration energy can be transmitted by vibration. Fig. 4(b) describes that the change of the loss factor has little effect on the reactive power flow curve, and with the increase of the loss factor, the peak of the reactive power flow reduced near the resonant frequency, which has little effect on the reactive power flow in other frequency ranges.

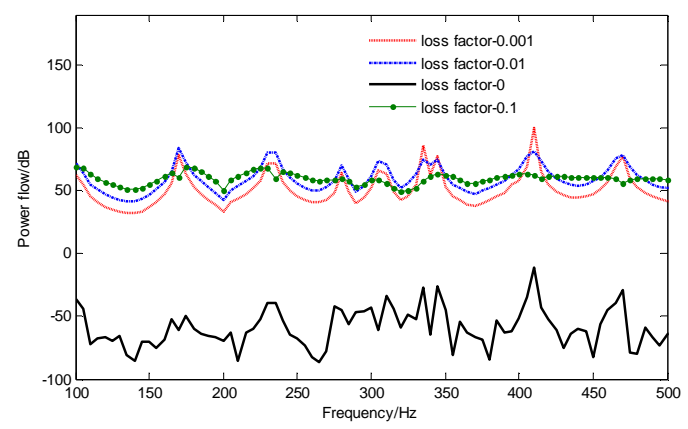

Fig. 4 Power flow with loss factor $\eta=0,0.1,0.001$.

The mechanical model of wave motion of Fig. 4 is consistent with the model of Fig. 4. The power flow curve is present in Fig. 4. With the increase of the loss factor, the peak of the power flow curve becomes narrow and steep, the corresponding frequency range is relatively narrow near the resonance peak. With the increase of loss factor, the vibration power flow decreases when the frequency away from the resonance range. The change of loss factor almost has no effect on the position of the resonance peak of power flow. When the loss factor is zero, the vibrational power flow is smaller than $-50 \mathrm{~dB}$ in frequency range, it is small enough to be ignored. The increase of loss factor could reduce the vibration energy only in resonance area, inversely, could increase the vibration energy away from resonance region. Also, loss factor couldn't change the resonance frequency. Vibration energy are almost from power flow peak at the resonance peak, so the increase 
of loss factor can reduce the transmission of vibration energy. The change of the loss factor almost has no effect on position at resonance peak of power flow.

\section{Conclusions}

In this paper, the steady-state response formulas of coupled plates were deduced by current MRRM with local dual coordinates. The direct inverse of MRRM was used to compute the dynamic response by self-programmed MATLAB. The MRRM results were compared with finite element numerical solutions, the correctness of the derivation of the MRRM formula was verified. The results of analysis of coupled plates are summarized as follows:

The local dual coordinate was introduced to MRRM, so the expression has a clear physical meaning, therefore the different boundary conditions of the elastic wave scattering and transmission of the physical relationship could be directly understood. After the local dual coordinates was introduced, the scattering matrix at the node is a constant matrix, which reduces the complexity of the formula derivation of coupled plates with much number of discrete plates.

The MRRM is a semi-analytical method, which could be an effective method of active vibration control. Through power flow analysis, the loss factor is verified to have a significant effect on the active power flow, the active power flow with no loss factor is much smaller than the active power flow. The loss factor has little effect on the reactive power flow, which could effectively reduce the peak value of the reactive power flow at the resonance frequency, but has little effect on the peak value of the reactive power flow in other frequency range. The increase of loss factor could reduce the vibration power flow only in resonance area, loss factor have the opposite effect on active power flow at resonance region and non-resonant region, and almost has no effect on position of power flow at resonance frequency.

\section{References:}

[1] Howard S M, Pao Y H. Analysis and experiments on stress waves in planar trusses [J]. Journal of Engineering Mechanics, 1998, 124(8):884-891.

[2] Cuschieri J M, McCollum M D. In-plane and out of plane waves' power transmission through L-plate junction using the mobility power flow approach [J]. The Journal of the Acoustical Society of America, 1996, 100(2):857-870.

[3] Kessissoglou, N J., Jie Pan. An analytical investigation of the active attenuation of the plate flexural wave transmission through a reinforcing beam [J]. The Journal of the Acoustical Society of America, 1997, 102(6):3530-3541.

[4] Kessissoglou N J. Power transmission in L-shaped plates including flexural and in-plane vibration [J]. Journal of the Acoustical Society of America, 2004, 115(3):1157-1169.

[5] Miao F X, Sun G, Chen K, et al. Reverberation-ray matrix analysis of the transient dynamic responses of asymmetrically laminated composite beams based on the first-order shear deformation theory [J]. Composite Structures, 2015, 119:394-411.

[6] Miao F X, Sun G, Chen K. Transient response analysis of balanced laminated composite beams by the method of reverberation-ray matrix [J]. International Journal of Mechanical Sciences, 2013, 77:121-129.

[7] Jiang J Q, Chen W Q. Reverberation-ray analysis of moving or distributive loads on a non-uniform elastic bar [J]. Journal of Sound and Vibration, 2009, 319(1):320-334.

[8] Liu C C, Li F, Huang W. Active vibration control of finite L-shaped beam with travelling wave approach [J]. Acta Mechanica Solida Sinica, 2010, 23(5):377-385.

[9] Liu C C, Li F M, Fang B, et al. The wave and vibratory power transmission in a finite L-shaped 
Mindlin plate with two simply supported opposite edges [J]. Acta Mechanica Sinica, 2011, 27(5):785-795.

[10] Liu C C, Li F M, Fang B, et al. Active control of power flow transmission in finite connected plate [J]. Journal of Sound \& Vibration, 2010, 329(20):4124-4135. 